\title{
The Effect of Color and Positional Noise on Reading Performance in Human Vision
}

\author{
Abdullah Alsalhi ${ }^{1,2}$, Nadia Northway ${ }^{1}$, Glyn Walsh ${ }^{1}$, Abd Elaziz Mohamed Elmadina $^{2 *}$ \\ ${ }^{1}$ Department of Vision Sciences, Glasgow Caledonian University, Glasgow, UK; ${ }^{2}$ Department of Optometry, Qassim University, \\ Qassim, Saudi Arabia
}

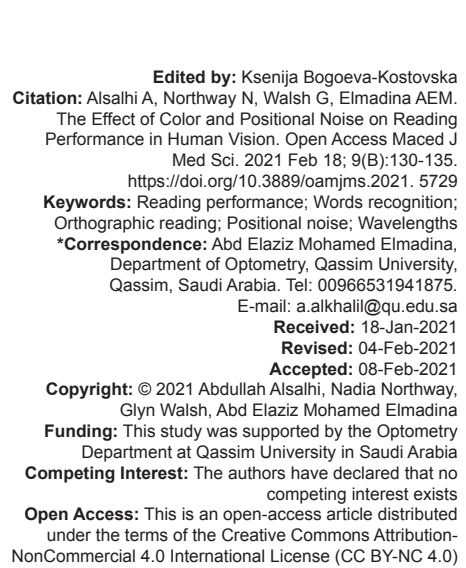

\section{Introduction}

Reading can be described as a complex cognitive process of decryption signs to create meaning. It is also a way of language achievement, communication, and sharing information and ideas. Therefore, reading is a basic requirement for an advanced society. There are three basic processes underlying reading: Sight perception, printed word recognition, and language comprehension [1]. Word recognition is considered a fundamental literacy skill that enables access to and processing of written language, as well as influencing reading performance. Word recognition can be described as the ability to accurately and automatically recognize words, with and without semantic context. It is a stamp of skilled readers' performance or the ability to accurately identify printed words [2].

Changing lighting and color are known to improve visual comfort and the perceptual difficulties that affect reading for those with poor vision [3], [4]. The role of colors in reading has been researched for many decades. It was mentioned back in 1958 that a student with reading difficulties was unable to recognize words printed on white paper but was able to recognize words printed on yellow paper [3]. It has been argued that colored overlays applied above written texts positively influence both reading fluency and reading speed [3]. Colored lenses and lighting are used to maximize visual efficiency in a range of patient groups such as those with reading difficulties. However, we do not know which wavelengths or colors can improve reading performance.

Introducing noise to measure human visual performance may provide important insights into the neural mechanisms (cortical processes) and computations used to solve a visual task [5]. There are some factors that affect reading performance by creating visual noise. One of the main factors that affect reading performance is letter spacing [6], [7], [8]. Manipulating the letter spacing can reduce the reading rate. According to Chung (2000), the reading rate increased with letter spacing, up to a critical letter spacing, and then, it either remained constant at the same reading speed or decreased slightly for larger letter spacing. The rate of the critical letter spacing was very close to the standard letter spacing and this held for all eccentricities (peripheral vision) and for both print sizes [6]. 
In human eyes, photoreceptors are the first link to visual perception, functioning as photon detectors. There are two types of photoreceptors: Rods and cones. Cones activate in the ranges of mid to bright light intensity. Cones are responsible for color vision as subtypes of cones are maximally sensitive to different wavelengths of light [9], [10]. There are three types of cones according to the wavelength of peak absorption: Short wavelength-sensitive cones (S-cones, max $\approx 420 \mathrm{~nm}$ ), which account for approximately $8-10 \%$ of the total number of cones; middle wavelength-sensitive cones (M-cones, $\max \approx 530 \mathrm{~nm}$ ), and long wavelengthsensitive cones (L-cones, max $\approx 560 \mathrm{~nm}$ ) [11], [12]. This study investigated the effect of changing the wavelength (along with types of cones by choosing three different wavelengths: Short, medium, and long) with different levels of positional noise on reading performance for normal vision participants. It also studied how this affects orthographic reading and the word recognition of real words. We measured the reading speed, duration time for reading, and the error percentage when changing the wavelength and level of positional noise.

\section{Methodology}

An interventional cross-section study included 20 English speakers (native and non-native) with bestcorrected distant visual acuity (BCVA) of $6 / 6$ or better participants (13 males and 7 females) (age range 18-38 years and mean age 28.9 years). The stimuli were presented in printed format. Stimuli were black print words in a horizontal arrangement on matte white card. The text samples contained unrelated words of $3,4,5$, and 6 characters, presented in 9 lines using Courier monospaced font, using black words on a white background. The distance between two adjacent words was two character widths, and the interline distance was five character heights. A simple random sample was selected from a set of the 560 most commonly used words in the English language [13] and was not repeated within the same trial. The viewing distance was $40 \mathrm{~cm}$ (Figure 1). The words had an optimal font size of 12 pt. (angular character size of $0.3 \mathrm{deg}$., defined as center-to-center spacing of horizontally adjacent characters) with contrast $(>90 \%)$.

First, words were degraded using positional noise produced by random vertical displacements of the letter position below or above the horizontal line on three levels. Each vertical letter position was sampled from a Gaussian distribution with zero mean and variance in the range 0.00 (N0), 0.30 (N1), and 0.60 $(\mathrm{N} 2) \times$ character height $^{2}$ (Figure 2).

Second, the wavelengths were changed from short to long stimuli. We used blue lighting (454 nm, short), green lighting $(514 \mathrm{~nm}$, mid), red

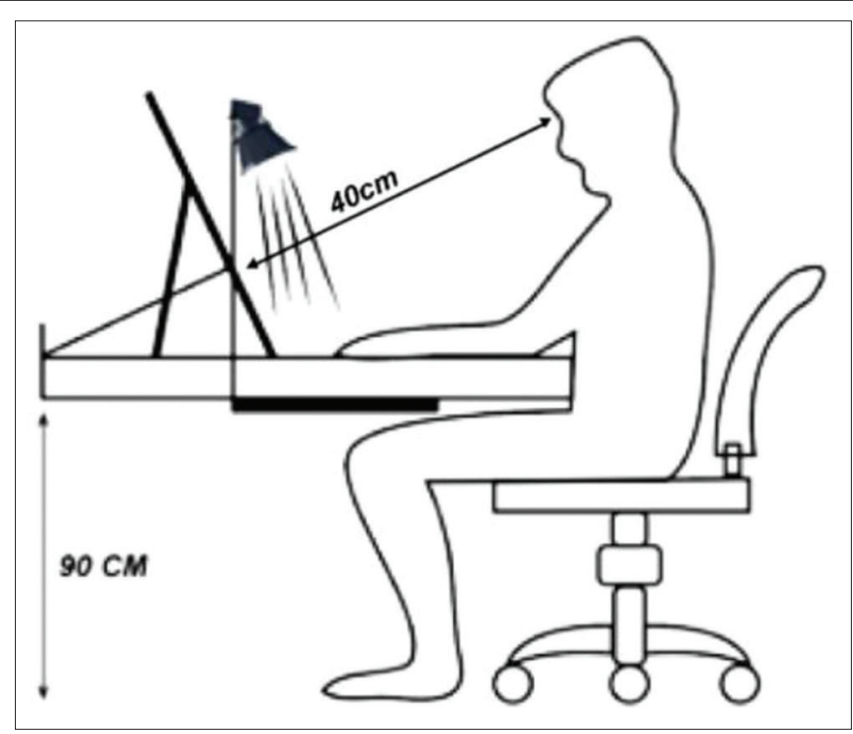

Figure 1: Experimental condition

lighting (620 nm, long), and white lighting as everyday lighting, with constant illumination for all four different wavelengths (30 lux).

The wavelengths were measured and controlled using a UPRtek spectrometer (MK350N Plus). The range of wavelengths measurable by the spectrometer (MK350N) is between $380 \mathrm{~nm}$ and 780 $\mathrm{nm}$.

All participants gave informed consent to take part in this study, which was approved by the Glasgow Caledonian University Ethics Committee. The participants were instructed to read the text samples aloud as fast as possible. They were given a brief explanation of all the experimental conditions. Tests with real words were presented in a random order. The participants were video recorded while they were reading out loud. The total number of words read and the number of words read incorrectly were counted. The participants were asked to read under three different colored LED lighting conditions: Short (blue), mid (green), and long (red) wavelength. White LED light (combining the same LED sources) was also used to more closely approximate normal reading lighting conditions. They were asked to read three different texts of real words with N0, N1, and N2. The participants were asked to read each text for $1 \mathrm{~min}$ under each wavelength.

\section{Results}

The Shapiro-Wilk and D'Agostino test indicated normal distribution of the data $(p>0.05)$ which fulfilled or satisfied the most important condition for using parametric tests ( $t$ and $F$ ) which were used in the GraphPad Prism program to analyze the data. 


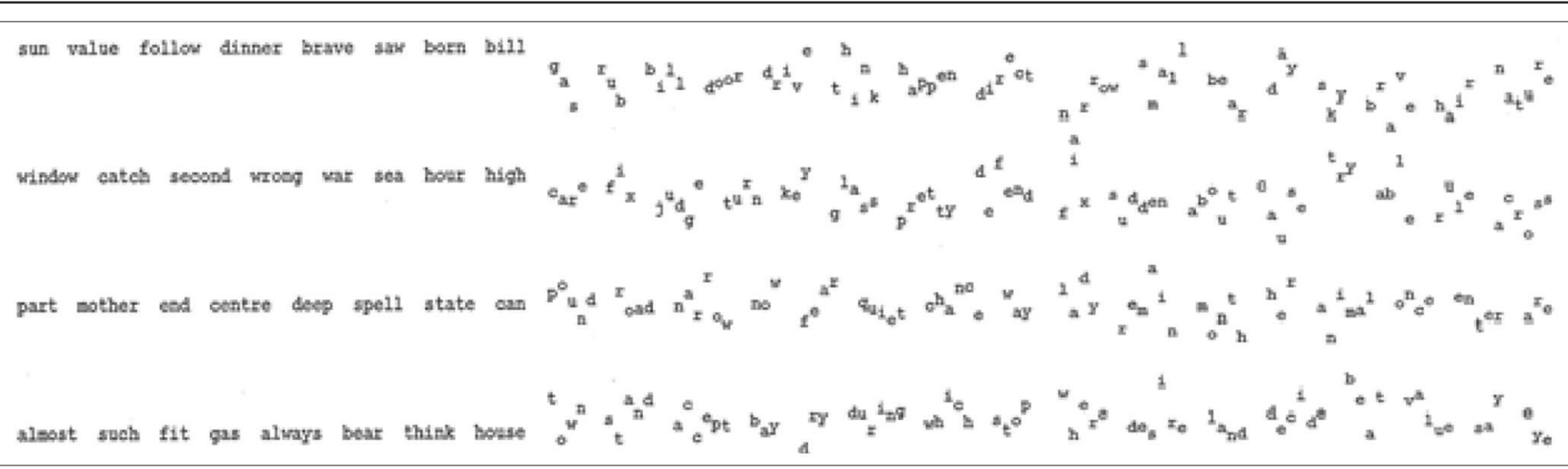

Figure 2: Sample of reading materials

We grouped the average reading rates for all the participants under the four different wavelengths and colors and calculated the average word per minute rate. The reading rate did not vary with the changes in wavelength, except that with the long wavelength (red), it was slower than with the other wavelengths, but the difference was not statistically significant (one-way ANOVA, $p=0.628, F=0.69$, and $R^{2}=0.026$ ) (Figure 3 ). We, therefore, calculated the change in reading rate for each individual to see what effect changing the wavelength had on their reading rate. We then calculated the difference for the wavelength (long, mid, and short) from the white wavelength (considered as mean). The results showed that the mean difference was reduced when the wavelength changed from long (red) to mid (green) or short (blue): It reduced by an average of 14 words per minute. This change was statistically significant (one-way ANOVA, $p<0.0001, F=4.2$, and $R^{2}$ $=0.53$ ). . However, the mean difference was not significant between the mid and short wavelengths, as it reduced by an average of 1.9 words per min (Figure 4$)(p=0.714)$.

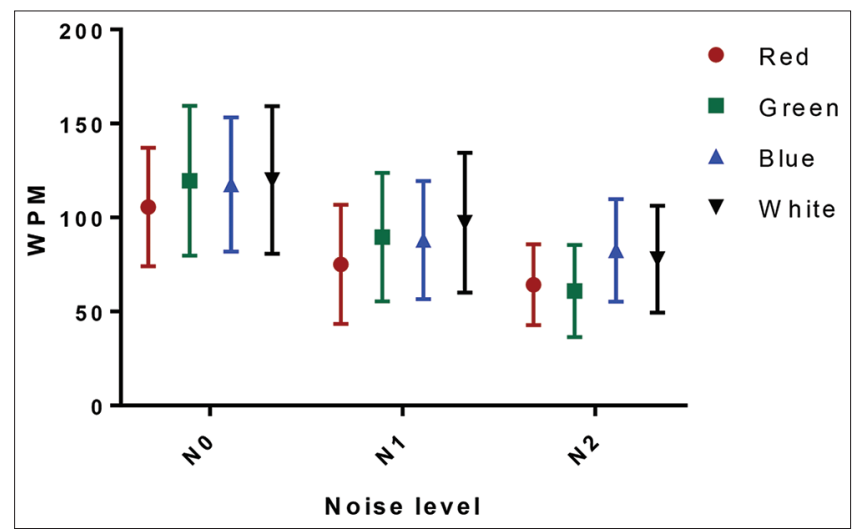

Figure 3: Mean reading rate $\pm S D$ under different wavelength and noise $(N)$

When introducing the positional noise, the average reading rate was recorded for all the participants under the four different colors and the three different levels of positional noise by calculating the average word per minute rate (Figure 3). The statistical analysis showed that the reading rate was not significantly reduced in any wavelength when introducing positional noise between N0 and N1 (one-way ANOVA, $p=0.147$ ). However, the

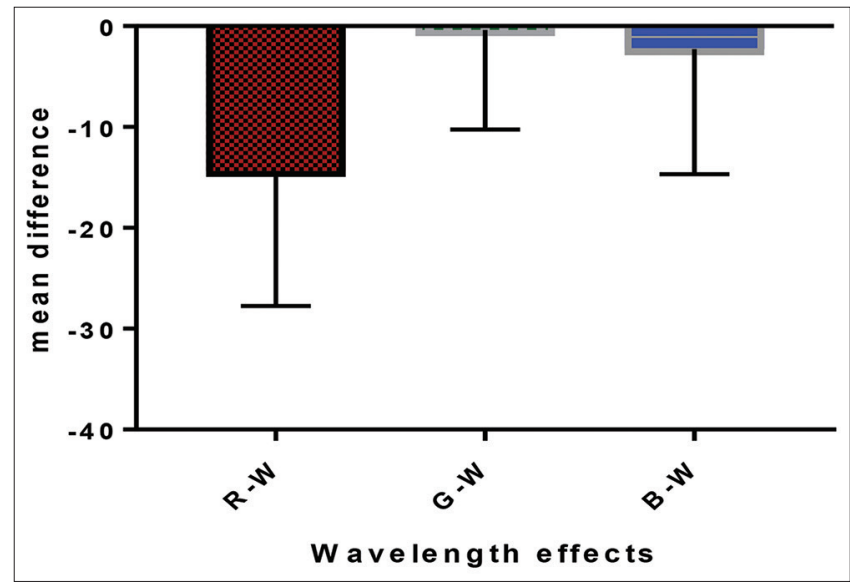

Figure 4: Mean differences in reading rate $\pm S D$ with in different wavelengths with NO

reading rate was significantly reduced when introducing positional noise between N0 and N2 (one-way ANOVA, $p<0.0001, F=8.3$, and $R^{2}=0.28$ ), but the changes with the short wavelength and white color were not significant (Figure 3). Furthermore, the statistical analysis suggested that the mean reduction in reading rate relative to white light when the wavelength changed from short to long or mid was significant, reducing by an average of 22 words per minute (one-way ANOVA, $p<0.0001, F=15$, and $R^{2}=0.35$ ). However, there was no significance in the mean difference between long and mid wavelength with positional noise, as the reading rate reduced by an average of 3.3 words per minute $(p=0.714)$ (Figure 5).

The linear regression tests were conducted on each color with and without positional noise. The reading rate as a function of positional noise was fitted with linear function. Furthermore, the reading performance was reduced when introduction the positional noise in all wavelengths (Figure 6). However, the initial reading (reading at 0 level of positional noise) was not similar with different wavelengths. The initial reading rates with short, mid wavelengths and white color were almost the same, but with long wavelength were slower compared with other colors. Moreover, a comparison of regressions in the different wavelengths revealed that the reduction in the reading rate was not similar for all the participants as the noise increased. In short wavelength, the gradient was the slower (the slope was flatter) compared with 


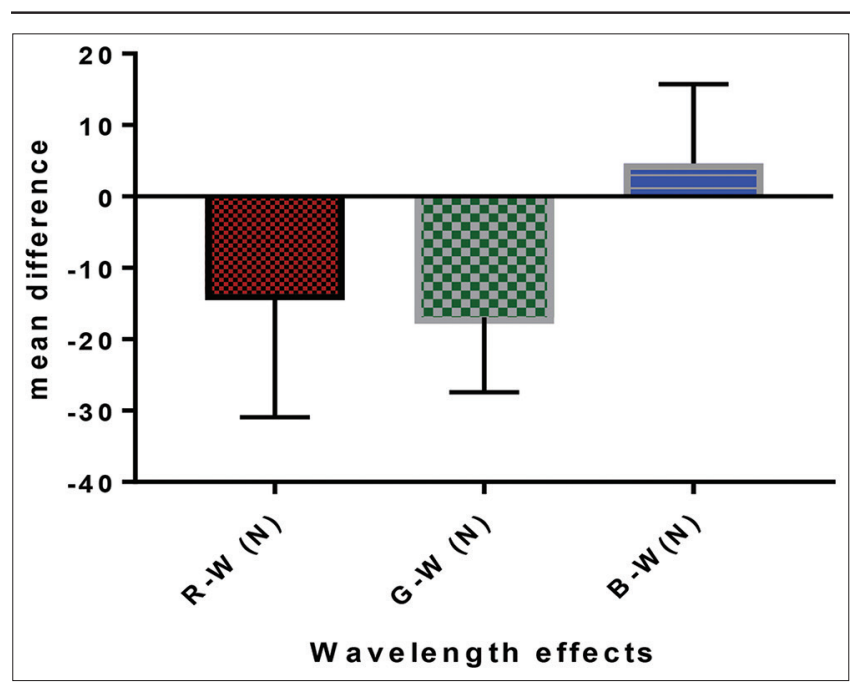

Figure 5: Mean differences in reading rate $\pm S D$ with in different wavelengths with noise

other wavelengths, and the mid wavelength was the highest gradient (the slope was steeper). The statistical analysis showed significant reading reduction with and without positional noise in mid (green) wavelength and white light $\left(p=0.0085, F=460\right.$, and $R^{2}=0.99$, which indicate $95 \%$ confidence intervals). However, the statistical analysis showed that there was no significance in the reading reduction for long (red) and short (blue) wavelengths $\left(p=0.169, F=13.3\right.$, and $R^{2}=0.93$, which indicate $95 \%$ confidence intervals). A comparison of regressions in the different wavelengths revealed that the reduction in reading rate was not similar for all the participants as the noise increased (Figure 6).

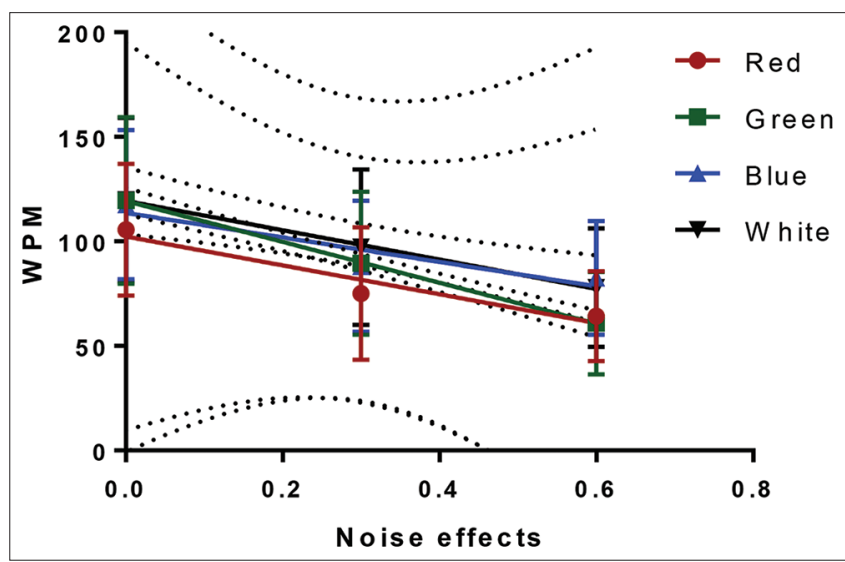

Figure 6: Linear regression function of reading rate with positional noise under different wavelengths

We analyzed the reading rate as a linear function of positional noise and the equations are as follows:

$$
\begin{array}{ll}
\text { 1. } & \text { Red }\left(Y=-69^{*} X+102.3\right) \\
\text { 2. } & \text { Green }\left(Y=-97.75^{*} X+119.3\right) \\
\text { 3. } & \text { Blue }\left(Y=-58.58^{*} X+113.6\right) \\
\text { 4. } & \text { White }\left(Y=-70.17^{*} X+119.4\right)
\end{array}
$$

The error rate was calculated as a percentage for each individual across the wavelength conditions. The error rate was calculated by dividing the number of incorrectly read words by the number of words read and multiplying by 100 . The error rate was similar across all conditions of different wavelengths for the same level of noise and statistically the differences were not significant. Furthermore, the error rate was increased when changing the noise level and statistical analysis revealed no significant difference between $\mathrm{NO}$ and $\mathrm{N} 1$ or N0 and N2 (one-way ANOVA, $p=0.185, F=4$, and $\left.\mathrm{R}^{2}=0.16\right)$ (Figure 7). Furthermore, the average time

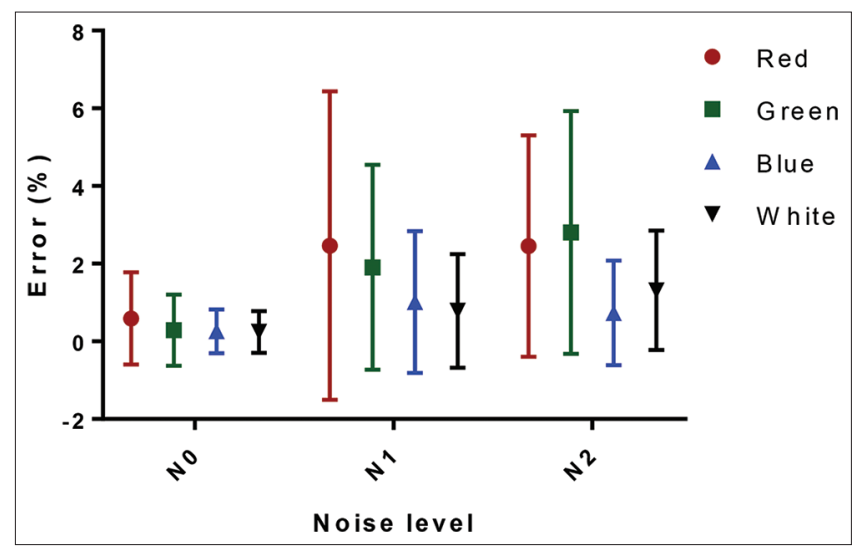

Figure 7: Mean reading error rate $\pm S D$ under different wavelengths and noise $(N)$

taken for each individual was calculated by dividing the number of words read by the time used $(60 \mathrm{~s})$. The duration of word fixation was unchanged across the light levels with the same level of noise (Figure 8). Furthermore, the average time increased when the noise level was changed for all levels of light but the statistical analysis showed no significant difference between NO and $\mathrm{N} 1$ for all wavelengths. However, the time for word fixation increased and there was a significant difference between $\mathrm{N} 0$ and $\mathrm{N} 2$ for long and mid wavelengths (onewayANOVA, $p<0.0001, F=7.1$, and $R^{2}=0.25$ ). However, the short and white wavelengths were not significant for the change between N0 and N2 ( $p=0.404)$.

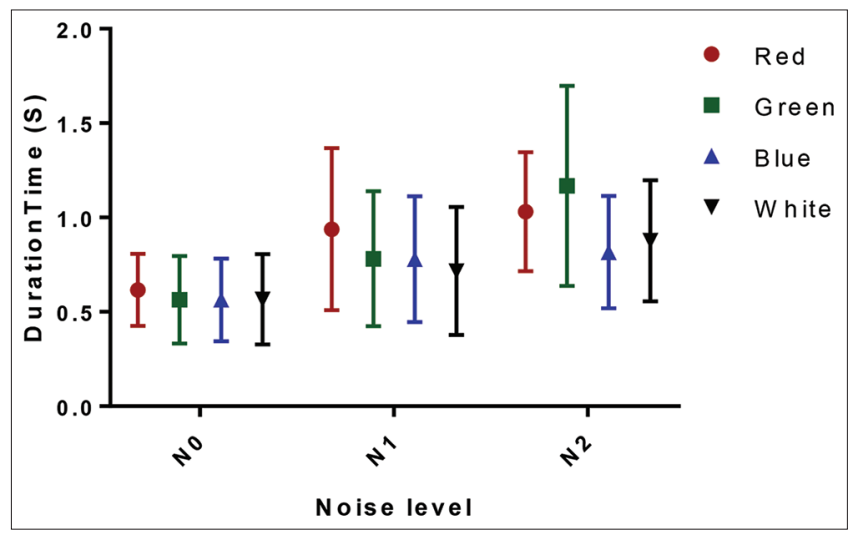

Figure 8: Mean duration time rate $\pm S D$ under different wavelengths and noise $(N)$

\section{Discussion}

This study has shown that reading speed was not affected by changing the wavelengths or color for 
the short and mid wavelengths and white color. The reading rate under a long wavelength (red) was the slowest but the results showed that the difference was not significant, compared with the other light conditions for readers with BCVA of 6/6 or better. This differs from what we expected based on the results of the previous studies, which reported the effects of overlay colors on reading performance [4], [14], [15]. However, the mean differences in wpm were reduced from the long to the mid and short wavelengths, but they were minimized between the mid and short wavelengths, which are also different to what we expected. This may be because of the role of magnocellular processing in reading performance, which reduces or impairs reading under red light (long wavelength) compared with normal (white) light [16].

When introducing positional noise, the results showed that reading speed was not significantly affected by changing the wavelengths or by introducing positional noise (N1). However, the introduction of positional noise (N2) significantly reduced reading speed under long and mid wavelengths. These results were expected because we found the same in a previous study with changing levels of noise. Furthermore, these results confirm that the color or wavelength of light affects reading performance and word recognition [4], [15]. This means that the different wavelengths and colors, especially short wavelength and white color, have different cortical effects by reducing the effects of positional noise on orthographic reading and word recognition. Moreover, binocular vision may reduce the noise effects or enhance orthographic reading, as has been shown by Murav'eva et al. [17], but this enhancement is not clear with different wavelengths or colors. Therefore, changing the wavelength and color produces cortical effects on word recognition and reading performance.

The mean differences in reading rate were reduced from short to long and short to mid wavelengths. However, positional noise had no effect with a short wavelength, which is different to what we expected. The magnocellular system is also believed to be enhanced by blue light and it allows rapid word identification, known as orthographic reading [16]. In addition, the previous studies have reported that koniocellular layers have been shown to transport short wavelength-sensitive cone signals to cytochrome-oxidase blobs in V1 in the cortex [18], [19]. The results provide evidence to support the belief that short wavelength (blue light) background colors have an impact on word recognition and reading performance by reducing the effects of positional noise.

In this study, the results showed that the reading rate decreased with green and white wavelengths as a linear function for positional noise levels. However, the reading rate function was not obviously different between the blue and red wavelengths, but the red wavelength produced the slowest rates of reading, compared with other light conditions as a linear function for positional noise levels. These results were unexpected. In the mid wavelength, the reading reduction seems to be a normal reduction because there is no factor affecting the reduction under the mid wavelength (green light). In the short wavelength, it is presumably because of the role of the $\mathrm{M}$ and $\mathrm{K}$ pathways, enhancing orthographic reading and word recognition by reducing the effect of positional noise [16], [19]. For long wavelengths, we would also have expected a significant reduction in reading rate because of the role of the $M$ pathway function and positional noise, impairing word recognition and reading performance [16].

This study demonstrated that the detrimental effects of positional noise on reading rate were less evident when the words were viewed in blue wavelength or short wavelength conditions. One possible explanation for this may be that only about $10 \%$ of cones process blue light [11], [12]. This may reduce the noise effects by processing through a small number of cones, reducing the amount of internal noise generated by the positional noise within the visual system. Alternatively, the blue light may enhance the orthographic ability of the reader by enhancing the magnocellular layers (M pathway) and koniocellular layers ( $\mathrm{K}$ pathway) function, enabling more rapid identification of the spatially disrupted words [12], [19]. Breitmeyer and Breier (1994) reported that the reaction time for the $\mathrm{M}$ pathway in stimulus was slower with a red background and faster with blue only for the target [20]. This means that the cortical processing is different with different wavelengths, which leads to effects on word recognition and reading performance. Furthermore, this is evidence that eye movement is unlikely to be a factor that affects word recognition [21], [22], [23].

This study showed that the error rate was not varied across all wavelengths when the level of positional noise was zero (N0). Furthermore, when changing the level of noise (N1 and N2), the error rate was not obviously different for any wavelengths. This was unexpected. It may because in this study, we used simple and short words and also single words rather than sentences, which enhances orthographic reading and word recognition [24].

The duration time for reading a word (fixation time) was unchanged across the different wavelengths with the same level of noise. However, average duration time was increased when changing the noise level for all levels of light and the statistical analysis showed no obvious differences between N0 and N1, and obvious differences between N0 and N2. However, fixation time was not significantly different between NO and N2 under the short wavelength, which is what we expected. This is presumably because of the role of the $\mathrm{M}$ and $\mathrm{K}$ pathways, enhancing orthographic reading and word recognition by reducing the effect of positional noise [16], [19]. Limitation of the current study mainly due to lighting was a bit higher than the threshold lighting for reading (25 lux), further cohort studies need to be conducted with more participants. 


\section{Conclusion}

The reading rate is not affected by changing the wavelength of the light. However, the mean differences in wpm were affected by changing the wavelengths. Furthermore, introducing positional noise affects word recognition differently with different wavelengths. The role of short wavelength in enhancing orthographic reading and word recognition is clear - they reduce the effects of positional noise. The error rate and duration time have different effects with different wavelengths, even when positional noise is introduced as well. How this may be correlated to the role of reading processes.

\section{Acknowledgment}

We would like to thank the Optometry Department at Qassim University in Saudi Arabia for sponsorship of this study. Furthermore, we would like to express our gratitude to the participants who have given their time willingly to participate in our research.

\section{References}

1. Keith R, Reichle ED. Models of the reading process: Wiley interdisciplinary reviews. Cogn Sci. 2010;1(6):787-99. https:// doi.org/10.1002/wcs.68

2. Latisha H, Flanigan K. Developing Word Recognition. New York: Guilford Publications; 2014. p. 1-24. Availale from: https://www guilford.com/excerpts/hayes4.pdf?t. [Last accessed on 2020 Nov 15].

3. Nadia N, Manahilov V, Simpson W. Coloured filters improve exclusion of perceptual noise in visually symptomatic dyslexics. J Res Reading. 2010;33(3):223-30. https://doi. org/10.1111/j.1467-9817.2009.01409.x

4. Arcangelo U, Enna M, Mulatti C. Colors, colored overlays, and reading skills. Front Psychol. 2014;5:1-4. https://doi. org/10.3389/fpsyg.2014.00833

\section{PMId:25120525}

5. Dennis ML, Klein SA, Chen I. What limits performance in the amblyopic visual system: Seeing signals in noise with an amblyopic brain. J Vision. 2008;8(4):1-23. https://doi.org/10.1167/8.4.1 PMId: 18484840

6. Susana TL. The effect of letter spacing on reading speed in central and peripheral vision. Investig Ophthalmol Vis Sci. 2002;43(4):1270-6.

PMId: 11923275

7. Veronica M, Facoetti A, Zorzi M. The effect of decreased interletter spacing on orthographic processing. Psychon Bull Rev. 2015;22(3):824-32. https://doi.org/10.3758/s13423-014-0728-9 PMId:25361820

8. Manuel P, Panadero V, Moret-Tatay C, Gómez P. The effects of inter-letter spacing in visual-word recognition: Evidence with young normal readers and developmental dyslexics.
Learn Instr. 2012;22(6):420-30. https://doi.org/10.1016/j. learninstruc.2012.04.001

9. Sheila AB, Kerov V. Photoreceptor Inner and Outer Segments Curr Top Membr. 2013;72:231-65.

PMId:24210432

10. Kolb H, Fernandez E, Nelson R. Webvision; 2012. Available from: http://www.iar.unicamp.br/lab/luz/ld/Pesquisa/webvision. pdf. [Last accessed on 2020 Nov 15]

11. Christina J. Human retinal circuitry and physiology. Psychol Neurosci. 2008;1(2):141-65

12. Helga K. How the retina works: Much of the construction of an image takes place in the retina itself through the use of specialized neural circuits. Am Sci. 2003;91(1):28-35.

13. Gordon EL, Yuanchao G. Stereopsis and contrast Vision Res. 1989;29(8):989-1004. https://doi org/10.1016/0042-6989(89)90114-4 PMId:2629214

14. Luz R, Bigham JP. Good background colours for readers: A study of people with and without dyslexia. Proceedings of the $19^{\text {th }}$ International ACM SIGACCESS Conference on Computers and Accessibility. 2017. p. 72-80. https://doi. org/10.1145/3132525.3132546

15. Catherine MS, Lawrenson JG, Conway ML. Efficacy of coloured overlays and lenses for treating reading difficulty: An overview of systematic reviews. Clin Exp Optom. 2018;101(4):514-20. https://doi.org/10.1111/cxo.12676

PMld:29633383

16. Christopher C, Ashourzadeh A, Kelly C, Monfette S, Kinsey K. Can the magnocellular pathway read? Evidence from studies of color. Vision Res. 2003;43(10):1211-22. https://doi.org/10.1016/ s0042-6989(03)00085-3

PMId:12705960

17. Murav'eva SV, Pronin SV, Vakhrameeva O, Shelepin $Y$ Comparing monocular and binocular visual acuity under noisy conditions. J Opt Technol. 2015;82(10):663-6. https://doi. org/10.1364/jot.82.000663

18. Stewart HC, Reid RC. The koniocellular pathway in primate vision. Ann Rev Neurosci. 2000;23(1):127-53.

PMId:10845061

19. Damian JW, Fitzpatrick D, Kerr JN. Primate thalamus: More than meets an eye. Curr Biol. 2016;26(2):R60-1. https://doi. org/10.1016/j.cub.2015.11.025 PMId:26811887

20. Bruno GB, Breier JI. Effects of background color on reaction time to stimuli varying in size and contrast: Inferences about human M channels. Vision Res. 1994;34(8):1039-45. https://doi. org/10.1016/0042-6989(94)90008-6 PMId:8160413

21. Reinhold K, Grabner E, Rolfs M, Engbert R. Length, frequency, and predictability effects of words on eye movements in reading. Eur J Cogn Psychol. 2004;16(1-2):262-84. https://doi. org/10.1080/09541440340000213

22. Helena $O$, Näsänen $R$, Kojo $I$. Eye movements in the visual search of word lists. Vision Res. 2002;42(12):1499-512. https:// doi.org/10.1016/s0042-6989(02)00077-9

PMId:12044751

23. Simon PT, Schroeder S. Word length and frequency effects on children's eye movements during silent reading. Vision $\begin{array}{lll}\text { Res. } \quad 2015 ; 113(\mathrm{Pt} & \text { A):33-43. https://doi.org/10.1016/j. }\end{array}$ visres.2015.05.008 PMId:26048684

24. Gerald T, Turcotte J. Word length effects are not due to proactive interference. Memory 2000;10(2):139-49:

PMId:11798443 